\title{
Single-dose Container
}

National Cancer Institute

\section{Source}

National Cancer Institute. Single-dose Container. NCI Thesaurus. Code C149896.

A container that holds a quantity of the preparation intended for total or partial use on one occasion only. 